\title{
Illegal Link Tracker
}

\author{
Vrushali Bongirwar ${ }^{*}$, Anjusha Pimpalshende ${ }^{2}$, Shailendra Aote ${ }^{3}$ and Archana Potnurwar ${ }^{4}$ \\ ${ }^{1,3}$ Shri Ramdeobaba College of Engineering and Management, Nagpur, India \\ ${ }^{2}$ CMR College of Engineering \& Technology, Hyderabad, India. \\ ${ }^{4}$ Priyadarshini Institute of Engineering \&t \&Technology, Nagpur, India
}

\section{ABSTRACT}

Most of the times, we encounter at situations, where we simply click on some links. This is because those links looks similar as the original links. The confidential information is stealing by using cyber attacks. Fraud websites may come to us, where we fail to differentiate it with original websites as images and logos looks similar. In this paper, Data mining techniques is proposed to detect phishing websites. A model is designed, which is used to classify the data. This model is then used for predicting whether the website is legal or illegal.

KEY WORDS: PHISHING, DATA MINING, ILLEGAL LINK TRACKER.

\section{INTRODUCTION}

In the current cyber world, the practice of phishing increases. Phishing is a kind of fraud where the attacker steals the important information of users such as login credentials, bank passwords. This information is gathered by sending mail, messages or any other communication medium. Victim receives a message, which appears as an important message to the users. These messages contain malicious software targeting user's account, which contains link that actually reads all the information from the user's machine. This information may also be taken from users camera or browsers. Phishing is very popular amongst attacker, because it is very easy to fool the people those always look for popular EMI's, sale, offers etc. Awareness amongst people is the main reason behind increasing the phishing practises.

\section{ARTICLE INFORMATION}

Received 10th Oct 2020 Accepted after revision 26th Dec 2020 Print ISSN: 0974-6455 Online ISSN: 2321-4007 CODEN: BBRCBA

Thomson Reuters ISI Web of Science Clarivate Analytics USA and Crossref Indexed Journal

\section{Clarivate
Analytics}

NAAS Journal Score 2020 (4.31) SJIF: 2020 (7.728)

A Society of Science and Nature Publication,

Bhopal India 2020. All rights reserved.

Online Contents Available at: http//www.bbrc.in/

Doi: http://dx.doi.org/10.21786/bbrc/13.14/19
Here, the aim is to design an application that will ensure safety from fake websites that is forwarded everyday at a huge scale. The application running in the background will alert user whenever the phone's web browser accesses any hoax link. The proposed system focuses on many factors such as: To control web browser from accessing fake websites; Before accessing any website, the app will ask permission to scan it so that user is safe; To ensure safety by securing personal information of user; Security is very important. Personal information will be secured so that his/her browsing experience is safe; To avoid leak of user data to phishing artists; User data information will be safe so that user does not become a victim of phishing attack.

Review of Literature: An illegitimate link tracker checks a link based on various parameters. This system revolves around checking these parameters and classifying on basis of them. The more are the parameters considered, more is the accuracy and efficiency of results. Alerting system should allow the user to request or cancel the emergency services in situation of false positives. https://www.psafe. com is website that helps to prevent phishing attacks and identifies a phishing link. Dfndr have 300+ million installations. "dfndr" security is a comprehensive tool goes beyond being simple antivirus software and helps protect your mobile privacy as well as enhancing your Android device's performance. New tools are constantly being added and upgraded to keep dfndr security cutting

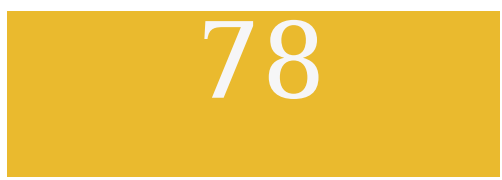


edge in an ever-evolving world of cyber threats. We continue to expand our product portfolio with apps such as dfndr vault, dfndr performance, dfndr vpn, and dfndr battery that safeguard and enhance Android and iOS users' experience. Limitation in existing systems are

1. Upgrading and maintenance of this system is very expensive process.

2. The main issue with this systemis that the notifications are sent to a web server and responders needs to check the web server for notification.

3. The system is not handy for mobile users and is time consuming as one needs to open the site and then access it.

\section{METHODOLOGY}

The procedure for implementation and a brief explanation of why it will be useful for implementing the proposed system is included, and a brief description of the current system development approach is counted here. The figure is an activity flow diagram of given system. The system focuses on three modules as showing in above figure, i.e.

\section{Feature Extraction}

2. Training Phase

3. Classification as spam or non-spam

Apart from these, the first decision block acts like a filter for trigger, if the user clicks on 'Abort' emergency services won't be alerted then. Else the nearest responder is selected for the providing the emergency service. And side by side, the location path is shown to the user and responder, both.

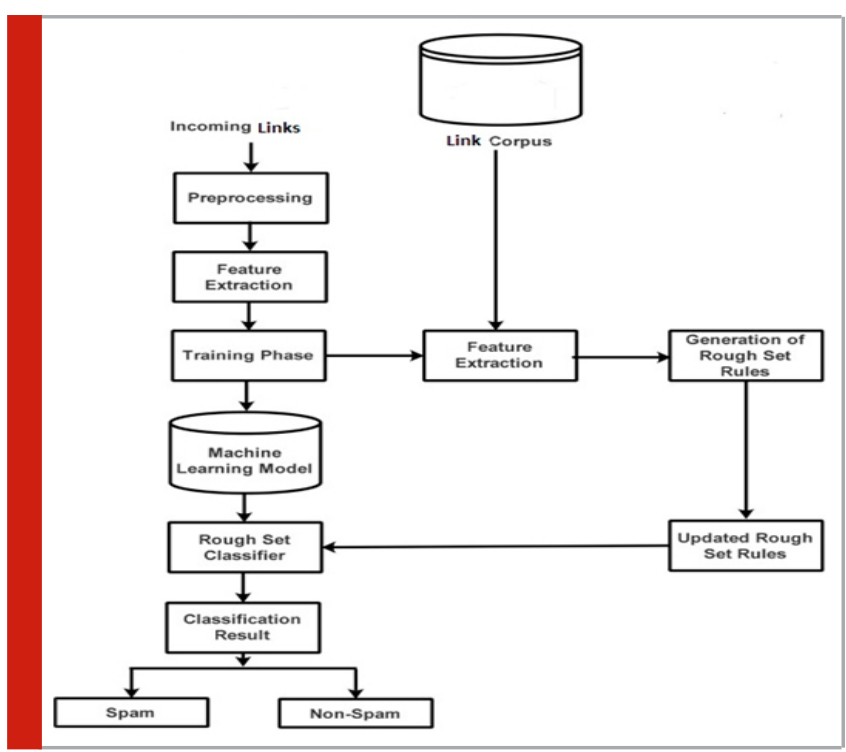

The dataset has a 30 predictors and the label is saved in the feature named Result. A value of 1 in the Result column denotes that the corresponding website is a phishing website and a value of -1 denotes that the corresponding website is a normal one.
Index(['having_IP_Address', 'URL_Length', 'Shortining_Service',

'having_At_Symbol', 'double_slash_redirecting', 'Prefix_Suffix',

having_Sub_Domain', 'SSLfinal_State', 'Domain_registeration_length',

'Favicon', 'port', 'HTTPS_token', 'Request_URL', 'URL_of_Anchor',

'Links_in_tags', 'SFH', 'Submitting_to_email', 'Abnormal_URL',

'Redirect', 'on mouseover', 'RightClick', 'popupwidnow', 'Iframe', 'age_of_domain', 'DNSRecord', 'web_traffic', 'Page_Rank',

'Google_Index', 'Links_pointing_to_page', 'Statistical_report', 'Result'],

\section{RESULT AND DISCUSSION}

Fake links and websites detection is implemented successfully. We have been able to identify whether a link is legitimate, illegitimate or suspicious based 15 different parameters. Random Forest Algorithm is used for classification as it has the best accuracy for our dataset over SVM or ID3. Android app is working in the front end and python codes run in the backend on Django server. This application is handy for all types of users and protects them from malicious sites which focus on phishing of data. Phishing of data is a scam worth millions of rupees per year. This application will prevent violation of privacy of user and safeguard their personal information and data from being mishandled.

The system accounts for improvisation of the accident alerting and benefits the user in many different ways. Moreover, the current system overpowers the existing system based on following crucial parameters:

1. Hardware independent: The current system is based on mobile based application and requires less or no dependency on external hardware, thus free from wear and tear.

2. Cost effective: The existing systems are costly and require quite maintenance as they are hardware based. Due to no such dependency in this system, accessibility to the application is quite cheap and easier.

3. Multiple factor consideration: The system takes various parameters as input. These parameters are calculated with the help of various existing and detected fake and trends observed in them. These parameters altogether make the detection and alerting mechanism effective and make less false predictions.

\section{CONCLUSION AND FUTURE SCOPE}

Now a days, number of evidences that the confidential information is stealing by using cyber attacks. Fraud websites may come to us, where we fail to differentiate it with original websites as images and logos looks similar. In this paper, a model based on data mining technique is proposed to classify the data. This model is then used for predicting whether the website is legal or Illegal. For future work, more progress is needed in research in order to detect the phishing links and aware the user about it. Adding additional feature such as, the application can be developed in such a way that will allow the user to report if the website if phishing which can be stored in database for future reference. More robust machine learning based models can be used to work up as classifier on the 
multiple set of attributes, which are promising. This will help to improve the accuracy of system.

\section{REFERENCES}

https://towardsdatascience.com/phishing-domaindetection-with-ml-5be9c99293e5

https://medium.com/backticks-tildes/lets-build-an-apiwith-django-rest-framework-32fcf40231e5 https://www.vinaysahni.com/best-practices-for-apragmatic-restful-api

https://stackabuse.com/random-forest-algorithm-withpython-and-scikit-learn/

https://androidclarified.com, retrofit-android-examplehttp-get-request

https://dataaspirant.com/2017/05/22/random-forestalgorithm-machine-learing/ 